\title{
Electrochemical behaviors of graphene decorated sulfurized polyurethane foam-based ultra-microporous carbon
}

\author{
Xianxian Zhou ${ }^{1}$, Min $\mathrm{Li}^{1}$, Chao Ge ${ }^{2, *}$, and Shibin $\mathrm{Liu}^{1, *}$ \\ ${ }^{1}$ College of Chemistry and Chemical Engineering, Taiyuan University of Technology, 030024 Taiyuan, P.R.China \\ ${ }^{2}$ College of Textile Engineering, Taiyuan University of Technology, 030600 Jinzhong, P.R.China
}

\begin{abstract}
Polyurethane foam is a typical commercial polymer with a large production quantity and its waste needs to be recycled. This work reports a facile method to prepare ultra-microporous carbon from the polyurethane foam (PUF) waste and use it as a reservoir to impregnate sulfur for Li-S batteries. The raw PUF was carbonized and then activated with $\mathrm{KOH}$. By controlling the activation temperatures, four carbon materials (PUFC-T) with different textures and N-doping levels were obtained. PUFC-800 shows the highest BET surface area, microporous volume, and quaternary $\mathrm{N}$ and pyridine $\mathrm{N}$-oxide species. The electrochemical test results showed that higher microporous volume is beneficial to increase the uniform distribution of sulfur, as a result, the cycle stability and rate capacity is improved significantly. A large reversible capacity of $542 \mathrm{mAh} \mathrm{g}^{-1}$ can be retained at a large current of $0.5 \mathrm{C}$ after 200 cycles with high sulfur loading of $70 \%$.
\end{abstract}

\section{Introduction}

With the increasing concern regarding the reduction of nonrenewable fossil fuels and the consequences of serious gadget population, more and more renewable and clean energy sources have been developed to replace the traditional energy materials.

Polyurethane foams (PUFs) are one of the most important thermoset polymers which were widely used in electric devices package. However, as a thermosetting polymer, polyurethane waste is quite difficult to be recycled. Consequently, most of them are directly burnt, causing severe environmental pollution as a result of giving off large amounts of nitrogen oxides and carbon oxides. [1-3] Actually, polyurethane foams wastes have the potential to prepare value-added nitrogen-doped carbon materials, which, suitable used in battery systems, additionally, can alleviate environmental pollution. [4-6]

Lithium-sulfur (Li-S) batteries have gained tremendous prospects as a candidate for energy storage devices, which in favor of the high theoretical specific capacity $\left(1670 \mathrm{~mA} \cdot \mathrm{h} \cdot \mathrm{g}^{-1}\right)$, high theoretical specific energy $\left(2675 \mathrm{~W} \cdot \mathrm{h} \cdot \mathrm{kg}^{-1}\right)$, low toxicity, and natural abundance of elemental sulfur. [7] However, its demerits such as poor conductivity of the sulfur and severe capacity degradation due to the soluble lithium polysulfides are still a big challenge. [8,9]

To address these issues, major efforts have been focused on the development of highly porous carbons with abundant surface functionalities and well-defined nanostructure primarily aiming to confine the sulfur and reduce the shuttling effect of polysulfides. Yang Hou et al. [10] presented a novel design of a lightweight carbon- based cathode by confining sulfur in 2D carbon nanosheets with abundant porous structure followed by 3D graphene aerogel wrapping, wherein porous carbon nanosheets act as the sulfur host and suppress the diffusion of polysulfide, while the graphene conductive networks anchor the sulfur-adsorbed carbon nanosheets, thus providing pathways for rapid electron/ion transport and preventing the polysulfide dissolution.

Furthermore, the nitrogen is introduced since the heteroatoms capture more strongly the lithium polysulfides through the coordination interaction between lithium polysulfides and heteroatoms. [11]

However, porous carbons suffer from a compromise between porosity, doped functionality, and nanostructure that have thus far restricted their performances.

Moreover, complex synthesis procedures and/or highly corrosive reagents were often employed to get porous carbon with improved electrochemical performance, which not only makes large-scale production impossible but also counteracts the cost advantage of Li-S batteries. The development of easily prepared carbon materials that capture effectively the soluble polysulfide intermediates with a large sulfur loading remains an engineering target.

Xiao et al. [4] reported a synthesis of nitrogen-doped porous carbon (NPC) from polyurethane foam (PUF) waste and use it as a reservoir to impregnate sulfur for Li-S batteries. The obtained NPC has a large surface area of $1315 \mathrm{~m}^{2} \cdot \mathrm{g}^{-1}$ with meso/micropores smaller than $5 \mathrm{~nm}$. Thermogravimetric analysis curves of NPC-S show that the sulfur contents are $75.2 \%$. The higher evaporation

* Corresponding author: gechao@tyut.edu.cn, sbliu@tyut.edu.cn 
temperature of sulfur in NPC-S was explained by more thermally stable in carbon support.

Polyurethane foam is obtained by nucleophilic addition reaction of polyether polyol and isocyanate. Because of the complexity of polyurethane foam structure, here, we report the design of ultra-microporous carbon derivatives from polyurethane foams waste by a simple chemical reagent active method. These carbon materials were sulfurized and graphene decorated, this composite was then used as a sulfur electrode in Li-S batteries. The effects of activation temperature on pore distribution, nitrogen content was discussed, and further, demonstrate their significance and effectiveness in enhancing the electrochemical performances.

\section{Experimental}

\subsection{Material preparation}

Schematic illustration showing the preparation process of Graphene-S/PUFC composite is presented in Fig. 1. The procedures for the preparation of ultramicroporous carbon with waste PUF used a $\mathrm{KOH}$ activation process of a previous study [5]. A certain amount of waste PUF (Instapak ${ }^{\circledR}$ packaging foams, Sealed Air company) was pre-carbonized at $400{ }^{\circ} \mathrm{C}$ for $1 \mathrm{~h}$ in a nitrogen flow (80 $\mathrm{mL} \cdot \mathrm{min}-1)$. This process could supply a graphitic precursor and better contact with $\mathrm{KOH}$. Then, the asobtained carbide products and $\mathrm{KOH}$ with a weight ratio of 1:2 were immersed into distilled water and stirred for $1 \mathrm{~h}$ at room temperature. After that, the black slurry was dried at $110{ }^{\circ} \mathrm{C}$ overnight to evaporate water. The dried sample was activated at $500-800{ }^{\circ} \mathrm{C}$ for $2 \mathrm{~h}$ in a nitrogen flow $(80 \mathrm{~mL} \cdot \mathrm{min}-1)$. Finally, the black solid was washed with $2 \mathrm{M} \mathrm{HCl}$ and deionized water until the filtrate became neutral. The obtained solid was dried at $110^{\circ} \mathrm{C}$ for $2-4 \mathrm{~h}$ and labeled as PUFC-T with $\mathrm{T}$ representing activation temperature $(\mathrm{T}=500,600,700$, $\left.800^{\circ} \mathrm{C}\right)$.

The S/PUFC-T composite was prepared by a simple melting infusion method. Specifically, a mixture of asprepared PUFC and elemental sulfur in a mass ratio of 3:7 was ground in ball milling for $90 \mathrm{~min}$, and then sealed in a Teflon container and heated to $155^{\circ} \mathrm{C}$ for 10 $\mathrm{h}$ under argon atmosphere. After cooling down to room temperature, four corresponding S/PUFC-T (labeled as S/PUFC-500, S/PUFC-600, S/PUFC-700, S/PUFC-800) composites were obtained.

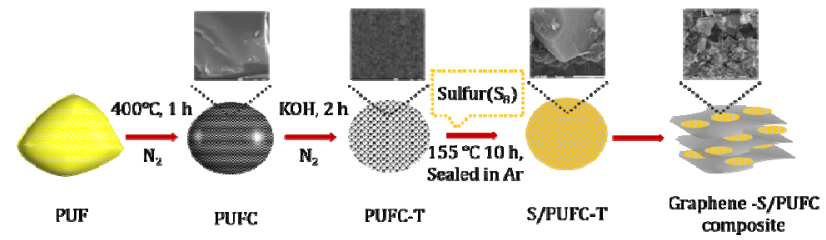

Fig. 1. Schematic illustration of the preparation processes of Graphene-S/PUFC-T composites

\subsection{Materials characterizations}

The morphologies and structures of the obtained samples were characterized through a scanning electron microscope (SEM, Quanta FEG 250, Japan), transmission electron microscopy (TEM, JEM-2100F, Japan), and X-ray diffraction (XRD, DX-2700 SSC $40 \mathrm{kV} / 30 \mathrm{~mA}$ ).

The specific surface area, total pore volume, and pore diameter distribution were determined from N2 adsorption-desorption isotherms, using a Surface Area and Porosity Analyzer (ASAP 2020HD88). Elemental analysis was performed on an Elemntar Vario EL Cube microanalyzer. Surface elemental analysis was accomplished by using an X-ray photoelectron spectrometer (XPS, K-alpha 1063). Digital photographs were taken with a Nikon COOLPIX s4300 Camera.

Sulfur loading in the S/PUFC-500, S/PUFC-800, composites were measured by a thermogravimetric analyzer (TGA, SDTQ600) in a nitrogen atmosphere with a heating rate of $10^{\circ} \mathrm{C} \cdot \mathrm{min}-1$ from 30 to $600{ }^{\circ} \mathrm{C}$.

\subsection{Electrochemical measurements}

To prepare the cathode for the lithium-sulfur battery, S/PUFC-T composites were mixed with graphene, and polyvinylidene fluoride in a mass ratio of 8:1:1 in NMP as dispersing solvent. The slurry was coated on aluminum foil ( $30 \mu \mathrm{m}$ in thickness) using a doctor blade and dried in a vacuum oven at $50{ }^{\circ} \mathrm{C}$ for $12 \mathrm{~h}$. The loading density of sulfur was about $1.2 \mathrm{mg} \cdot \mathrm{cm}-2$. CR2025-type coin cells were assembled using the prepared cathode with lithium foil as the counter electrode. Celgard 2400 was used as the separator. The electrolyte used was $1 \mathrm{M}$ Lithium bis(trifluoromethan sulfonyl) imide in tetra (ethylene glycol) dimethyl ether and 1,3-dioxolane $(1: 1 \mathrm{~V} / \mathrm{V})$ containing $1 \%$ LiNO3. The electrolyte volume of $60 \mu \mathrm{L}$ was injected into the CR2025 coin cell. Galvanostatic cycling was carried out using a battery tester (LAND) from 1.6-2.8 $\mathrm{V}$ versus $\mathrm{Li}+/ \mathrm{Li}$ at $0.2 \mathrm{C}$ and $0.5 \mathrm{C}(1 \mathrm{C}=1675 \mathrm{mAh} \cdot \mathrm{g}-1)$. Cyclic voltammetry $(\mathrm{CV})$ measurements were performed on a multichannel electrochemical workstation (CHI1030C) at a scan rate of $0.1 \mathrm{mV} / \mathrm{s}$. The rate performance was evaluated at $0.1,0.2,0.5,1$, and $2 \mathrm{C}$. Electrochemical Impedance Spectroscopy (EIS) measurements were performed using a multichannel electrochemical workstation (CHI1006E) within the frequency range of $100 \mathrm{kHz}$ to $0.01 \mathrm{~Hz}$ at amplitudes of $10 \mathrm{mV}$.

\section{Results and discussion}

\subsection{The physical characterization of the PUFC samples}

Fig. 2 list the photograph of PUF (a) and SEM (b-e) and TEM images (f) of PUFC. PUF is obtained from Instapak ${ }^{\circledR}$ packaging foams, one of high performance, lightweight cushioning, blocking and bracing, and void fill materials. In our work, the PUF was a soft foam with a light yellow color.

Fig. 2(b-e) shows SEM images of PUFC under different activation temperatures $\left(500,600,700,800^{\circ} \mathrm{C}\right)$. 
It is seen that all of the samples have a typical porous carbon structure with a rough surface. Along with the increase of activation temperature, more carbon was reacted with $\mathrm{KOH}$, as it can be seen the pore diameter increased and form an interconnected pore network that obtained hierarchical pores. Specifically, a large number of micropores together with some macro/mesopores distributed in the carbon framework are visible from the TEM image (Fig. 2f).

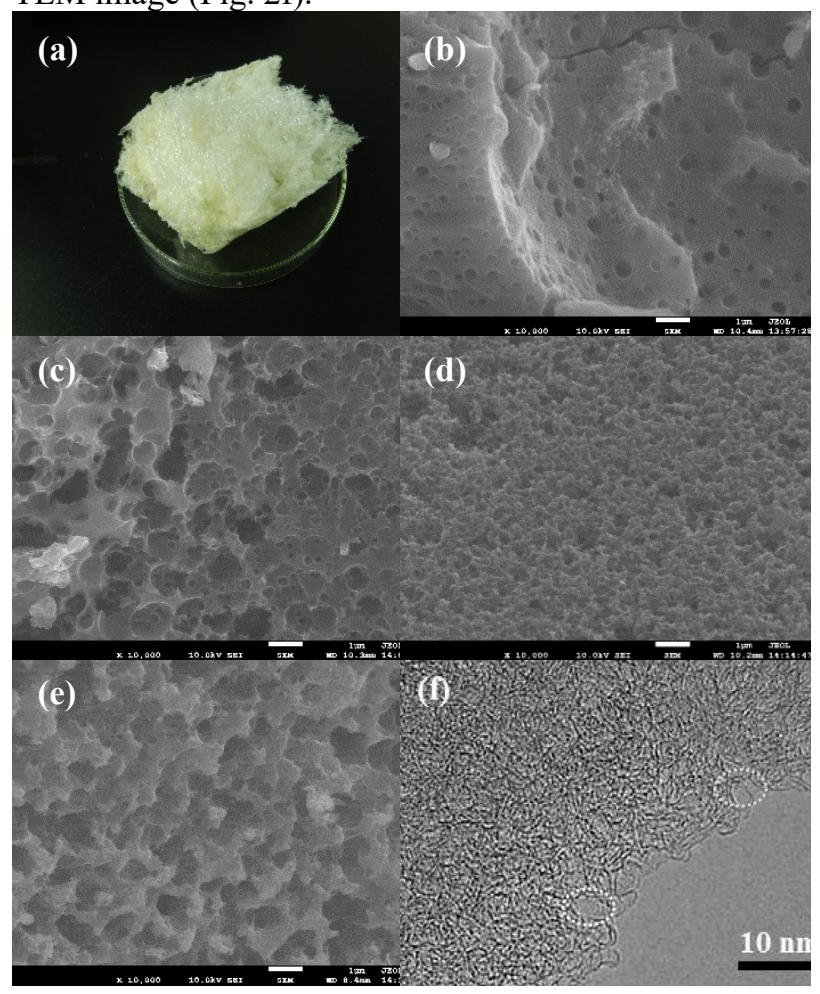

Fig.2. photograph(a), Scanning electron microscope(b-e) and tansmission electron microscope(f) morphologies of PUFC-T $\left(\mathrm{T}=500,600,700,800^{\circ} \mathrm{C}\right)$

To clarify the pore structure of PUFC under different activation temperatures, the $\mathrm{N} 2$ adsorption /desorption isotherms, and the pore size distribution plots obtained using the density functional theory (DFT) method. The textural properties are listed in Table 1. Results showed that The PUFC with abundant ultra-micropores with a size of $0.7 \mathrm{~nm}$ could be prepared by $\mathrm{KOH}$ activation. Although the most probable pore diameter distribution was similar along with the increase of temperature, the BET surface area and total pore volume increased sharply with the increase of temperature. Fig 3(a) shows that the ratio of microporous volume to total pore volume was above $80 \%$ indicate the microporous carbon which derives from the $\mathrm{PUF}+\mathrm{KOH}$ activation method. These results indicate the sample of PUFC-800 as the sulfur hosting substrate would accommodate more sulfur especially small S2-4 molecules and apply more active sites. It can be also deduced that the shuttle problem could be avoided and good cycling stability is expected to be obtained because the micropores have strong physical force with polysulfides and also limit the polysulfides convert to cyclo-S8. The volume ratio of macro-mesopores increasing from $9 \%$ to $19 \%$ when temperature increased from 500 to $800{ }^{\circ} \mathrm{C}$, suggesting the sulfur hosting substrate would exhibit better rate performance along with the increasing of temperature with the similar sulfur content. This is because previous works suggested that the meso- and macropores contributed to the improvement of the electrolyte penetration and lithium-ion transfer rate. [11]
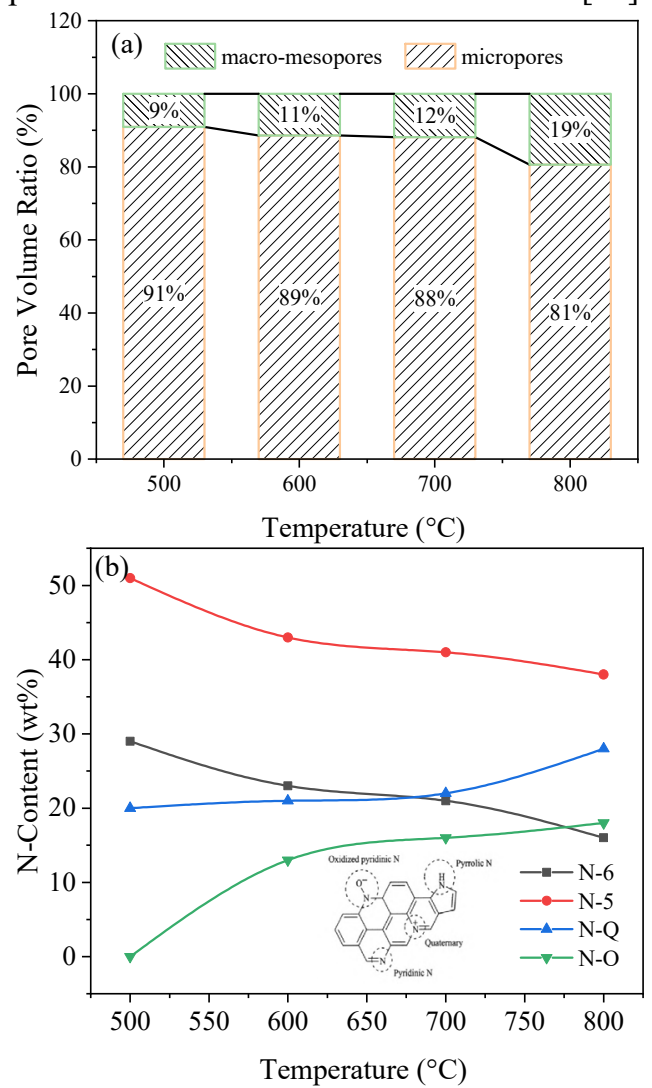

Fig.3. The ratio of microporous (a) and nitrogen content of PUFC-T $\left(\mathrm{T}=500,600,700,800^{\circ} \mathrm{C}\right)$

Table 1 also shows the element analysis result of PUFC, with the increasing activation temperature, nitrogen content decrease.

From the test result of $\mathrm{N} 1 \mathrm{~s}$ XPS of the PUF based carbon materials. The components at the binding energies of $398.6,399.9,401$, and $402.8 \mathrm{eV}$ are attributed to pyridinic $\mathrm{N}(\mathrm{N}-6)$, pyrrolic or pyridine $\mathrm{N}$ $(\mathrm{N}-5)$, quaternary or graphitic $\mathrm{N}(\mathrm{N}-\mathrm{Q})$, and pyridine $\mathrm{N}$ oxide species (N-O), respectively, in which the N-5 and $\mathrm{N}-6$ can provide the binding sites for polysulfides, whereas the N-Q is beneficial for enhancing the electrical conductivity of the carbon.[12-14]

Fig 3(b) shows the tendency of the four nitrogen along with the activation temperature. Pyridine N-oxide species were not detected in PUF-500; however, they were presented in PUF-600, 700, and 800, and their relative amount increased with the activation temperature. This is also held for the quaternary $\mathrm{N}$ species. In contrast, the relative contents of pyridinic and pyrrole-type nitrogen species gradually decreased from $29 \%$ and $51 \%$ to $16 \%$ and $38 \%$, respectively, when the activation temperature was increased from 500 to $800{ }^{\circ} \mathrm{C}$. This indicates that some of the pyridinic and pyrroletype nitrogen species are likely transformed into quaternary $\mathrm{N}$ and pyridine $\mathrm{N}$-oxide species at high activation temperature despite that the total amount of different types of nitrogen species decreased. 


\subsection{The physical characterization of the S-PUFC samples}

The as-prepared PUFC samples were further characterized by X-ray powder diffraction (XRD) to investigate their crystallinity state. As shown in Fig. 4 (a), the four samples exhibit a typical broad peak centered at $2 \theta \approx 23^{\circ}$ and a weak peak at $2 \theta \approx 43^{\circ}$, which are corresponded to the diffraction of $(002)$ and (101) planes of graphite, respectively. The weak intensity of the XRD peaks indicates the low degree of crystallinity and graphitization of these three samples, this is a typical character of the activated carbon. PUFC- 800 shows the strongest peak intensity at the diffraction of (101) planes which means a higher graphitized degree among the four samples. After loading S into the porous PUFC samples, the XRD patterns of the four composites are very similar. The signature peaks of elemental sulfur are observed indicating that sulfur is not only embedded in the pores but also much of them presented on the surface. The sulfur loading ability of the PUFC prepared under different temperature was calculated according to the sulfur density of $2.07 \mathrm{~g} \cdot \mathrm{cm}-3$, the pore volume value could afford a theoretical sulfur loading of $18.5 \mathrm{wt} \%$, $42.0 \mathrm{wt} \%, 55.0 \mathrm{wt} \%$, and $58.1 \mathrm{wt} \%$ in the pores (as shown in Table 1). In our experiment, the sulfur to carbon weight ratio was 7:3 for all of the samples, as a result, the sulfur contents were excessive and most of them were covered on the surface of the PUFC, especially for PUFC-500. However, PUFC-800 with a large BET surface area and pore volume will supply enough inner space to sulfur, as it can be seen from the thermogravimetric analysis. TG curves of the Sulfur, PUFC, and S/PUFC-500, 800 samples under the N2 atmosphere were carried out to assign the actual sulfur contents in the composites (Fig. 4c). it can be seen that very similar sulfur mass loading $(66-67 \%)$ in S/PUFC500 and S/PUFC-800 composites. The mass loss curve of S/PUFC-500 was similar to pure sulfur in the same experiment condition. However, for the S/PUFC-800 composite, the weight loss shows two steps, $45.21 \%$ under $300{ }^{\circ} \mathrm{C}$ and $21.75 \%$ in the temperature range of $300-450{ }^{\circ} \mathrm{C}$. The latter could be attributed to the loss of the small S2-4 accommodated in the ultra-micropores, while the former corresponds to the evaporation of the excessive sulfur, which beyond the pore accommodation capacity of the PUFC and exists as cyclo-S8 outside the carbon matrix.[11] The difference between the cyclo-S8 and the small S2-4 might result from the strong interaction between the carbon ultra-micropores and the sulfur [15] as well as the great confinement ability of the PUFC.

\subsection{The electrochemical performance of the S/PUFC cathode materials}

To study the influence of PUFC samples microstructures on the electrochemical performance of sulfur, the electrochemical behaviors of as-prepared S/PUFC-T composites are investigated by cyclic voltammogram (CV) and galvanostatic charge-discharge. CV curves of $\mathrm{S} / \mathrm{PUFC}$ cathodes with different porous textures were measured in the potential range of $1.6-2.8 \mathrm{~V}$ at a scan rate of $0.1 \mathrm{mV} \mathrm{s}-1$.
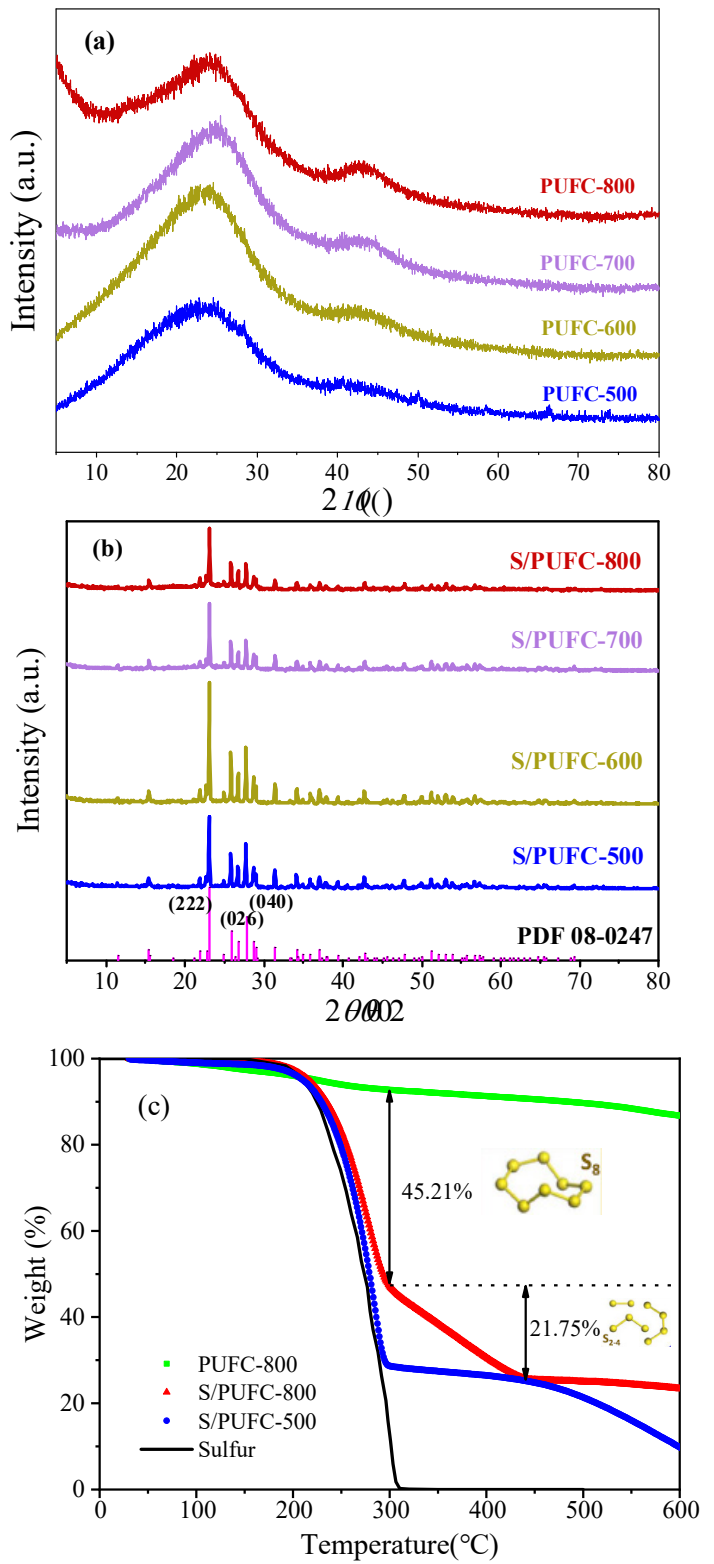

Fig.4. XRD patterns of (a) PUFC- $T$, (b)S/PUFC-T, $(T=500$, $\left.600,700,800^{\circ} \mathrm{C}\right)(\mathrm{c})$ thermogravimetric curves of samples

As shown in Figure 5 (a), only one oxidation peak at around $2.42-2.55 \mathrm{~V}$ is observed during the anode scan process of the four cathodes. Two reduction peaks at 2.26, and 1.70-2.09 $\mathrm{V}$ during the cathode scan process, which represents the transition from elemental $\mathrm{S}$ to the long-chain LiPSs, further the transition from the longchain LiPSs to Li2S2 or Li2S. Compared with CV curves of S/PUFC-500, S/PUFC-600,700, and 800 presented lower oxidation potential and higher reduction potential which represent the higher electrochemical reaction efficiency of the Li-S batteries.

Besides, the S/PUFC-800 cathode exhibits a larger peak area than that of the other three cathodes, which suggests the porous structural design for the sulfur host not only can increase the specific capacity of the Li-S batteries but also improve the utilization rate of the sulfur. The reduction peaks of the lower temperature cathode show a broader reduction curve, which can be 
attributed to an increase in the impedance with lower electricity and lower porosity of carbon material at $500{ }^{\circ} \mathrm{C}$.
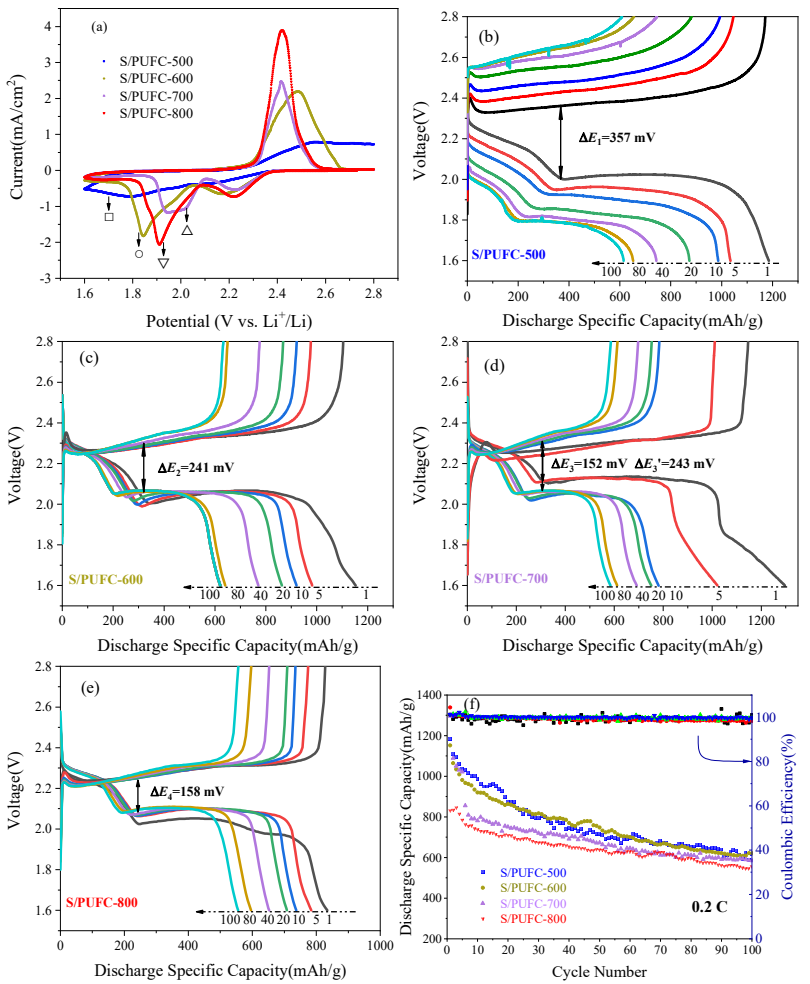

Fig.5. (a)CV curves at a scan rate of $0.1 \mathrm{mV} \mathrm{s}^{-1}$ in the first cycle. (b-e) discharge specific capacity (f) cyclic performance and coulombic efficiencies of S/PUFC-T ( $\mathrm{T}=500,600,700$, $\left.800^{\circ} \mathrm{C}\right)$

The discharge specific capacity of the S/PUFC-T composite electrodes are shown in Fig. 5(b-e). It is detected that there are two distinct discharge platforms at 2.3 and $2.1 \mathrm{~V}$ in the first cycle which were correspondence to the two initial reduction potentials in CV curves, respectively. The high voltage platform at $2.3 \mathrm{~V}$ is attributed to $\mathrm{S} 8$ reduce to high order $\mathrm{Li}$ organosulfur units (Li2Sx, $\mathrm{x} \geq 8$ ) and Li2S8. The slope between $2.1-2.3 \mathrm{~V}$ can be assigned to the formation of Li2Sx $(4 \leq x \leq 6)$. Upon further discharge, these Li2Sx species $(4 \leq \mathrm{x} \leq 6)$ is finally reduced to fully discharged $\mathrm{Li} 2 \mathrm{~S} 2 / \mathrm{Li} 2 \mathrm{~S}$ at a lower voltage platform at $2.1 \mathrm{~V}$.

However, by comparing Fig. 5(b) with (c-e), the discharge capacity and voltage platform of the S/PUFC500 electrode was sharply decreased from the first cycle to the 100th cycles. It is revealed that lower sulfur utilization and electric conductivity because of the lower porosity and graphitization in PUFC-500. Besides, the energy barrier $(\Delta \mathrm{E})$ of the charge-discharge curves decreased $357 \mathrm{mV}(\Delta \mathrm{E} 1)$ to $158 \mathrm{mV}(\Delta \mathrm{E} 4)$ along with the increase of the temperature. It is interestingly found that S/PUFC-700 shows a voltage drop and the energy barrier was increasing from $152 \mathrm{mV}$ to $243 \mathrm{mV}$ between the 5 th to the 10 th cycle. The two energy barriers were familiar with the S/PUFC-600 $(\Delta \mathrm{E} 2=241 \mathrm{mV})$ and S/PUFC-800 $(\Delta \mathrm{E} 4=158 \mathrm{mV})$ electrode. It is reflecting the limiting effect of the microstructure of the PUFC on LiPSs. Fig 5 (f) shows the cycling ability of the four electrodes. After the unavoidable capacity loss in the first few cycles, the S/PUFC-700 and S/PUFC-800 electrodes exhibit more stable cycling performance than the S/PUFC-500 and S/PUFC-600. It can be found the large amount of ultra-microporous is not benefiting for the initial capacity because of the small sulfur molecular was formed. The cyclic performance and coulombic efficiencies of the four electrodes are found to be 614.8mAh·g-1, $620.9 \mathrm{mAh} \cdot \mathrm{g}-1,584.1 \mathrm{mAh} \cdot \mathrm{g}-1,555.3$ $\mathrm{mAh} \cdot \mathrm{g}-1$ after 100 cycles at $0.2 \mathrm{C}$, retaining about $62.3 \%, 67.5 \%, 74.8 \%$, and $75.3 \%$ of the 10 th cycle capacities, respectively. The coulombic efficiency of the four electrodes slightly fluctuates around $100 \%$, indicating the outstanding reversibility. It can be deduced that the cycle stability would be increasing with the microporous volume because of the reduced polysulfide shuttle effect. This effect can be further elucidated by rate capability as shown in Fig 6 (a).
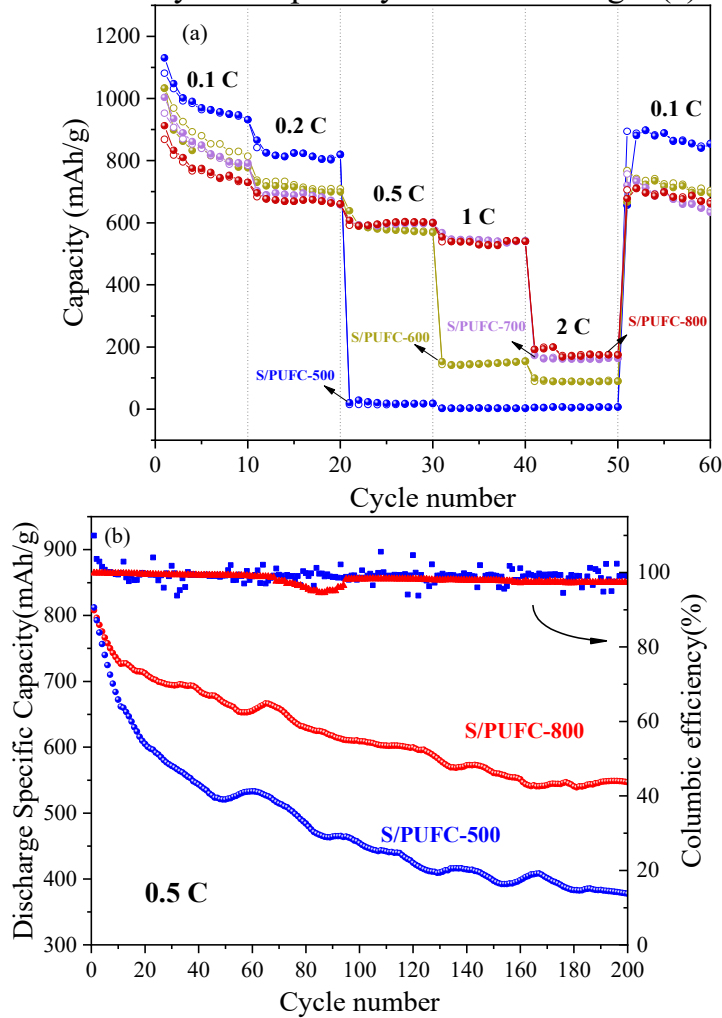

Fig.6. (a) Rate performance at various $\mathrm{C}$ rate and (b)cyclic performance and coulombic efficiencies of S/PUFC-T

The capability of the S/PUFC-800 cathode was compared with those of the other three cathodes at rates from 0.1 to $2 \mathrm{C}(1 \mathrm{C}=1675 \mathrm{mAh} \mathrm{g}-1)$, as shown in Figure $6 \mathrm{a}$. The S/PUFC-800 cathodes exhibited the best rate performance, with specific capacities of 912, 696, 608, 554, and $199 \mathrm{mAh} \mathrm{g-1}$ at $0.1,0.2,0.5,1$, and $2 \mathrm{C}$, respectively. The highly reversible and excellent rate performance of the S/PUFC-800 cathodes was further demonstrated by the easy recovery of the capacity after cycling. The longer cycling life of the $\mathrm{Li}-\mathrm{S}$ battery using the S/PUFC-500 and S/PUFC-800 cathode at $0.5 \mathrm{C}$ is demonstrated in Figure $6 \mathrm{~b}$. The S/PUFC-800 cathode exhibited better cycling durability. The discharge capacity remained at $542 \mathrm{mAh} \mathrm{g}-1$ after 200 cycles vs $762 \mathrm{mAh} \mathrm{g}-1$ at the first cycle, indicating a low capacity decay rate of $0.144 \%$ per cycle, which is comparable or better than that of other high-energy $\mathrm{Li}-\mathrm{S}$ batteries which derived from waste materials. 
Table 1. Comparison of physical properties and electrochemical performances of this work with reported carbonaceous materials at

\begin{tabular}{|c|c|c|c|c|c|c|}
\hline \multirow[t]{2}{*}{$\begin{array}{l}\text { Carbonaceous } \\
\text { materials }\end{array}$} & \multicolumn{2}{|c|}{ Textural properties } & \multirow{2}{*}{$\begin{array}{l}\mathrm{N} \\
\text { content } \\
(\mathrm{wt} \%) \\
\mathrm{N}\end{array}$} & \multicolumn{2}{|c|}{$\begin{array}{l}\text { S content in mass } \\
(\mathrm{wt} \%)\end{array}$} & \multirow[t]{2}{*}{ Performance $(\mathrm{mAh} / \mathrm{g})$} \\
\hline & $\begin{array}{l}\mathrm{S}_{\mathrm{BET}} \\
\left(\mathrm{m}^{2} \cdot \mathrm{g}^{-1}\right)\end{array}$ & $\begin{array}{l}\mathrm{V}_{\mathrm{t}} \\
\left(\mathrm{cm}^{3} \cdot \mathrm{g}^{-1}\right)\end{array}$ & & theory* & $\begin{array}{l}\text { exper- } \\
\text { iment }\end{array}$ & \\
\hline PUFC-500 & 169.1 & 0.11 & 9.03 & 18.5 & 66.9 & $0.2 \mathrm{C} 100$ cycle 614.8 \\
\hline PUFC-600 & 825.9 & 0.35 & 8.71 & 42.0 & 70 & $0.2 \mathrm{C} 100$ cycle 620.9 \\
\hline PUFC-700 & 1430.0 & 0.59 & 6.92 & 55.0 & 70 & $0.2 \mathrm{C} 100$ cycle 584.1 \\
\hline PUFC-800 & 1469.9 & 0.67 & 4.22 & 58.1 & 66.9 & $0.5 \mathrm{C} 200$ cycle 542 \\
\hline NPC(PUFF)[4] & 1315 & 0.76 & 7.18 & 61.1 & 75.2 & $0.1 \mathrm{C} 100$ cycle 766 \\
\hline NDPC-1(sugar cane bagasse) [11] & 2762.5 & 1.85 & 8.48 & 79.3 & 53 & $0.5 \mathrm{C} 400$ cycle 571 \\
\hline NOPC (Soybean residue) [16] & 2690.3 & 1.34 & - & 73.5 & 64.5 & $0.2 \mathrm{C} 100$ cycle 758 \\
\hline UMC-3(PVDF)[17] & 1062 & 0.45 & - & 48.2 & 55.8 & $\begin{array}{l}0.1 \mathrm{C} 100 \text { cycle } 626.3 \\
(1-3 \mathrm{~V})\end{array}$ \\
\hline
\end{tabular}

* Calculated according to the sulfur density of $2.07 \mathrm{~g} \cdot \mathrm{cm}^{-3}$

\section{Conclusion}

An ultra-microporous carbon derivative from polyurethane foams waste was produced by a simple chemical reagent active method. These carbon materials were sulfurized and graphene decorated and then used as a sulfur electrode in Li-S batteries. Results showed that the higher microporous volume and quaternary $\mathrm{N}$ and pyridine $\mathrm{N}$-oxide species are beneficial to increase the uniform distribution of sulfur and improve the cycle and rate performance. S/PUFC-800 composite delivers a large reversible capacity of $740.4 \mathrm{mAh} \cdot \mathrm{g}^{-1}$ with good cycling performances and excellent high-rate capabilities. A large reversible capacity of $542 \mathrm{mAh} \cdot \mathrm{g}^{-1}$ can be retained at $0.5 \mathrm{C}$ after 200 cycles. However, the large amount of ultra-microporous is not benefiting for the initial capacity because of the small sulfur molecular was formed.

We acknowledge the funding support from the Applied Basic Research Program of Shanxi Province (201901D211064, 201901D211061), the National Natural Science Foundation of China (Grant 21706171. No.21802101), the Key Research and Development Program of Shanxi Province (201803D121120).

\section{References}

1. J. O. Akindoyo, M. D. H. Beg, S. Ghazali, M. R. Islam, N. Jeyaratnam and A. R. Yuvaraj. RSC Advances. 6, 114453 (2016)

2. Y. Wang, W. Kang, C. Chen, X. Zhang, L. Yang, X. Chen, G. Cui, Y. Zhang, F. Zhang and S. Li. J. Hazard. Mater. 365, 395 (2019)
3. R. H. Krämer, M. Zammarano, G. T. Linteris, U. W. Gedde and J. W. Gilman. Polym. Degrad. Stab. 95, 1115 (2010)

4. S. Xiao, S. Liu, J. Zhang and Y. Wang. J. Power Sources. 293, 119 (2015)

5. C. Ge, J. Song, Z. Qin, J. Wang and W. Fan. ACS Applied Materials \& Interfaces. 8, 18849 (2016)

6. Z. X. Cao, J. Zhang, Y. M. Ding, Y. L. Li, M. J. Shi, H. Y. Yue, Y. Qiao, Y. H. Yin and S. T. Yang. J. Mater. Chem. A. 4, 8636 (2016)

7. S. Ruan, Z. Huang, W. Cai, C. Ma, X. Liu, J. Wang, W. Qiao and L. Ling. Chem. Eng. J. 385, 123840 (2020)

8. A. Kilic, Ç. Odabaşı, R. Yildirim and D. Eroglu. Chem. Eng. J. 390, 124117 (2020)

9. X. Liu, W. Huang, D. Wang, J. Tian and Z. Shan. J. Power Sources. 355, 211 (2017)

10. Y. Hou, J. Li, X. Gao, Z. Wen, C. Yuan and J. Chen. Nanoscale. 8, 8228 (2016)

11. S. Wang, K. Zou, Y. Qian, Y. Deng, L. Zhang and G. Chen. Carbon. 144, 745 (2019)

12. J. Yan, X. Liu, X. Wang and B. Li. Journal of Materials Chemistry A. 3, 10127 (2015)

13. G. Zhou, L.-C. Yin, D.-W. Wang, L. Li, S. Pei, I. R. Gentle, F. Li and H.-M. Cheng. ACS Nano. 7, 5367 (2013)

14. L. Ji, M. Rao, H. Zheng, L. Zhang, Y. Li, W. Duan, J. Guo, E. J. Cairns and Y. Zhang. J. Am. Chem. Soc. 133, 18522 (2011)

15. X.-J. Hong, X.-Y. Tang, Q. Wei, C.-L. Song, S.-Y. Wang, R.-F. Dong, Y.-P. Cai and L.-P. Si. ACS Applied Materials \& Interfaces. 10, 9435 (2018)

16. F. Chen, J. Yang, T. Bai, B. Long and X. Zhou. Electrochim. Acta. 192, 99 (2016) 
17. Q. Zhu, Q. Zhao, Y. An, B. Anasori, H. Wang and B.

Xu. Nano Energy. 33, 402 (2017) 